\title{
Sociologie néostructurale, disciplines sociales et systèmes complexes
}

\section{Emmanuel Lazega et Christophe Prieur}

\section{(2) OpenEdition \\ Journals}

Édition électronique

URL : http://journals.openedition.org/rsl/455

DOI : $10.4000 /$ rsl. 455

ISSN : 2271-6246

Éditeur

Éditions Rue d'Ulm

Référence électronique

Emmanuel Lazega et Christophe Prieur, « Sociologie néostructurale, disciplines sociales et systèmes complexes », Revue Sciences/Lettres [En ligne], 2 | 2014, mis en ligne le 07 octobre 2013, consulté le 19 avril 2019. URL : http://journals.openedition.org/rsl/455; DOI : 10.4000/rsl.455

Ce document a été généré automatiquement le 19 avril 2019

(c) Revue Sciences/Lettres 


\title{
Sociologie néostructurale, disciplines sociales et systèmes complexes
}

\author{
Emmanuel Lazega et Christophe Prieur
}

\section{Big surveillance}

1 Un fait divers comme on en recense beaucoup, à ranger dans la rubrique «big surveillance »: en mars 2012, l'Administration Obama prend la décision, controversée mais désormais en vigueur, de permettre à l'une des nouvelles agences américaines créées au lendemain du 11 septembre 2001, le National Counter-Terrorism Center, connue sous le nom de NCTC, de rassembler et de fusionner en une seule base de données toutes les informations dont dispose le gouvernement sur tout citoyen américain, qu'il soit ou non suspecté dans, ou lié à, une procédure d'enquête spécifique menée par les pouvoirs publics ${ }^{1}$.

2 Jusque-là, les bases de données des différents ministères et agences gouvernementales étaient officiellement séparées et supposées étanches. Pour les partisans de cette centralisation, il ne s'agit que d'efficacité technique en matière de lieu et de durée de conservation des données. Pour les opposants il s'agit de l'acquisition et de la mise en œuvre d'un pouvoir nouveau et organisé de surveillance des citoyens ${ }^{2}$ qui modifie irréversiblement les relations entre État et citoyens, voire entre État et grandes organisations privées (entreprises et associations) d'une part, citoyens individuels d'autre part. Les tentatives précédentes, par exemples celles des centres de recherche du Pentagone (dont un programme intitulé Total Information Awareness en 2002), avaient déjà préconisé - sans en obtenir le droit officiel - la concentration de toutes les bases de données privées et publiques existant aux États-Unis à des fins de recherche d'indices d'activité terroriste. Le NCTC procède désormais à toutes les analyses qu'il souhaite mener sur les individus, leurs comportements, leurs activités organisées, leurs réseaux, à partir des données dont disposent les pouvoirs publics aux États-Unis (dans les domaines financier, économique, scolaire, policier, de santé, relationnel, etc.). Il peut aussi partager 
et échanger des informations avec des gouvernements étrangers lorsqu'il le juge nécessaire. D'après les médias américains, cette décision politique, bien que controversée au sein même de l'Administration, n'a pas rencontré beaucoup de résistance.

3 L'une des nombreuses questions que pose cette évolution est liée à la nature nouvelle des données accumulées dans ces bases, par exemple les données de réseaux sociaux et organisationnels. Dans la mesure où les liens entre les pouvoirs publics (aux États-Unis) et les entreprises comme Facebook et Google sont notoires, «big surveillance » s'étend désormais non seulement à des individus mais à leurs réseaux sociaux et organisationnels ainsi qu'aux relations entre ces réseaux. Les institutions du type NCTC disposent déjà de données de ce type par le biais de la connaissance des affiliations familiales, institutionnelles, associatives ou professionnelles des citoyens. Comme en témoignent les récentes révélations par le Washington Post (7 juin 2013) sur le programme américain Prism de surveillance, par la National Security Agency, des internautes étrangers, elles accèdent systématiquement à des données riches sur les profils relationnels des individus tels qu'ils apparaissent dans leurs réseaux sociaux en ligne et leurs carnets d'adresses numériques. Mais au-delà de la surveillance des individus, dont la vie privée est bien entendu menacée, leur véritable objet est d'être capables de reconstituer, voire d'anticiper, l'émergence des différentes formes d'organisation sociale, de mobilisation et d'action collective de la société civile. Cela inclut la connaissance des dirigeants et des participants, de leur auto-organisation et techniques de diffusion de l'information dans les médias sociaux, les propriétés structurales et sémantiques de ces mouvements, leurs coordinations, capacités de mobilisation et ressources collectives, y compris leurs processus sociaux.

\section{Zooming-in, zooming out : l'émergence des « grands » réseaux riches}

4 Ce fait divers n'est qu'un indicateur parmi beaucoup d'autres de changements actuels considérables qui sont de grands défis pour les sociétés contemporaines, mais aussi pour les sciences sociales établies et les nouvelles disciplines qui envisagent d'intervenir dans l'étude du comportement social, comme les sciences dites de la « complexité » (incluant informaticiens, biologistes, neurologues, mathématiciens, physiciens statisticiens, entre autres). L'une des spécificités de ces dernières est l'analyse des très grands réseaux (des medias socionumériques comme la blogosphère, les groupes ou listes d'acteurs collectifs qui se «suivent» en temps réel, qui communiquent dans des environnements sociotechniques nouveaux, etc.). À l'échelle immense où ces nouvelles bases de données situent l'étude du comportement social, les méthodes utilisées par ces approches détectent par exemple des "communautés » de manière mécanique, y compris des dynamiques associées à ces «communautés ». Tout comme les praticiens du marketing ciblé cherchent des niches spécifiques et des intermédiaires-prescripteurs particulièrement centraux entre ces niches, l'analyse et la détection de communautés permet le zooming in et le zooming out dont, entre autres, la NCTC a besoin.

5 Jusqu'à présent, les très grands réseaux sociaux numériques étudiés étaient constitués d'un très grand nombre d'entités dont on savait souvent très peu de choses, et les relations entre ces entités sont d'ordinaire décrites, elles aussi, de manière très simplifiée : les « données » sont considérées à un niveau de généralité très élevé, suffisant 
pour identifier ces "communautés", mais rarement suffisant pour spécifier, par exemple, des formes différentes d'action collective ${ }^{3}$. L'internet, par exemple, est considéré comme un système de nœuds et d'interconnexions entre lesquelles circulent de l'information: on en sait peu sur la manière dont l'information que récupèrent les internautes sur internet est construite, ni de la manière dont elle circule sur le web pour se rendre disponible pour leurs requêtes (Guillaume et Latapy, 2005 ; Tarissan et al., 2009). Les théories de l'action sur lesquelles ces approches s'appuient, lorsqu'elles existent, sont minimales. Dans un livre récent, Albert-László Barabási (2003), liste des modèles mathématiques qui sont proposés par la physique statistique pour l'analyse des très grands réseaux, quels qu'ils soient. Il reconnaitt l'existence de régularités dans les comportements humains, des régularités qu'il pense pouvoir identifier, voire prédire avec les grands réseaux sociaux " pauvres ». La vision de l'acteur social qu'il propose pour cela, et donc pour observer et prédire des régularités, est la suivante : «Think of yourself as a dreaming robot on autopilot, and you will be much closer to the truth» (p.11).

Cette théorie de l'action minimale se pose des questions sur les réseaux sociaux comme un ensemble de problèmes théoriques de mathématiques pures. Ce qui n'est pas mathématiquement pur, relève du «terrain» et de ses applications. La complexité de l'abstraction mathématique, nécessaire pour faire progresser les méthodes, est différente de celle du "terrain", dont on a souvent besoin de s'abstraire pour avancer. La présentation de réseaux comme des "petits mondes» à invariance d'échelle, par exemple, s'appuie dans la grande majorité des cas sur un modèle d'accumulation des relations qui produit, notamment, une centralisation progressive sans turnover relationnel ni mécanisme inverse de décentralisation, comme on pourrait l'observer dans des systèmes concrets, sur le « terrain » de la vie sociale.

7 S'il ne fait aucun doute, à ce niveau de généralité, que des grands réseaux sociaux et des réseaux métaboliques, par exemple, peuvent partager des propriétés mathématiques formelles communes, la question de la nature des dynamiques communes à cette grande variété de réseaux reste ouverte. Mais au-delà, avec «big surveillance», l'approche centrée sur l'alliance entre "mathématiques pures » et "grand réseaux pauvres » est en passe de dater. Les grandes bases de données s'enrichissent suffisamment pour envisager la nécessité d'une convergence entre la complexité abstraite des mathématiques pures et la complexité du « terrain » sociologique. Et donc d'une collaboration entre représentants des sciences de la complexité, avec les sciences sociales.

\section{Sociologie néostructurale et complexité : les convergences actuelles}

8 C'est à ce stade que se repose la question de la nature du dialogue entre la sociologie néostructurale, plus familière de l'étude des petits réseaux riches, avec les disciplines engagées actuellement dans l'analyse des systèmes complexes. Entre les interactions microscopiques et le comportement global, la structure de niveau méso est très peu prise en compte dans la modélisation contemporaine des systèmes complexes, alors que c'est elle qui intéresse le plus les sociologues centrés sur une statistique des petits réseaux multiniveaux caractéristiques de la société organisationnelle et marchande. La conception des méthodes qui permettent aux sciences de la nature et aux sciences sociales de se développer conjointement en est encore à un stade très exploratoire. Mais surtout les épistémologies des uns et des autres sont très différentes. 
9 Sur le plan des méthodes, mais aussi de la connaissance substantive des phénomènes, les convergences sont déjà nombreuses et ne peuvent toutes être mentionnées ici. Par exemple, pour Chavalarias (2006), les activités des agents ne sont pas indexées à des critères donnés une fois pour toutes : l'émergence de structures et de différenciations sociales est liée à des processus de coévolution culturelle créant des liens entre des ensembles de motivations possibles. Pour Cointet et Roth (2007), ce sont davantage des modèles mathématiques calibrés et calés sur des données d'observation réalistes construites à partir d'une conception de l'acteur plus complexe au départ qui devraient être utilisés pour procéder aux simulations expérimentales des sciences de la complexité. Dans ses travaux empiriques sur les réseaux d'affiliation (2-mode), Latapy et al. (2008) s'inspirent des questions sociologiques qu'on peut se poser sur les terrains divers d'où proviennent leurs données.

10 Pour la sociologie, en particulier néostructurale qui travaille aussi sur des graphes, l'acteur social n'est pas un robot rêveur régulé par pilotage automatique car ces robots sont rarement confrontés aux dilemmes de l'action collective et aux grands débats micro, méso ou macropolitiques que ces dilemmes suscitent (justice, cohésion sociale, régulation, etc.). Pour cette sociologie, la complexité vient de ce que les acteurs n'agissent que s'ils donnent un sens et une pertinence à leur action. De plus ils sont capables, par l'apprentissage individuel, relationnel et collectif, de modifier ces significations, de réévaluer ces pertinences et de changer de comportements de manière stratégique. Ils le font enfin en tenant compte des actions des autres acteurs avec lesquels ils sont ou non en relation. Les processus sociaux qui émergent de ces interactions sous contrainte (les formes de solidarité, de contrôle, de régulation, etc.), sont parmi leurs principaux sujets de préoccupations politiques. Les règles qu'ils se donnent à eux-mêmes sont aussi importantes que les régularités qui peuvent s'observer "indépendamment " de leur travail politique, à supposer même que de telles régularités peuvent exister de manière séparée.

\section{Du côté du «terrain »: La société organisationnelle et ses processus de niveau mésosocial}

11 Ainsi, une autre raison de s'intéresser au fait divers de la NCTC réside dans le fait qu'il évoque le nouveau contexte dans lequel les processus "génériques » et bottom-up de l'organisation sociale (solidarités, contrôles, apprentissages, régulations, etc.) se déploient. En simplifiant à l'extrême, ce contexte est celui de la société « organisationnelle» (Perrow, 1991): une société caractérisée par la dépendance au salariat, l'externalisation des coûts sociaux de la production, l'«absorption » de la vie sociale par d'énormes appareils organisationnels et leurs satellites, ainsi que la conception et l'utilisation de l'action publique par des acteurs privés aux pouvoirs inédits.

Dans ce contexte, les formes de discipline sociale que les citoyens acceptent de considérer comme légitimes, c'est-à-dire les formes de discipline qui rendent possibles leurs actions collectives organisées, sont précisément bâties sur ces processus sociaux. Par discipline sociale nous entendons les formes de solidarité et d'exclusion, de socialisation et d'apprentissage, de contrôle social et de résolution de conflits, de régulation et d'institutionnalisation. La discipline sociale est faite de tous les processus sociaux qui permettent de faciliter la gestion des dilemmes de l'action collective. Cette dernière est 
toujours plus ou moins organisée et locale, i.e. spécifique aux lieux de vie, qu'ils soient de travail, familiaux, associatifs, marchands, de mobilisation politique, etc. C'est aussi sur la base de la connaissance, aussi bien abstraite que concrète, de cette discipline que les citoyens cherchent à évaluer la légitimité des structures dans lesquelles les pouvoirs mis en œuvre dans l'action sont distribués de manière asymétrique, souvent fortement concentrés, souvent exercés sans contre-pouvoirs.

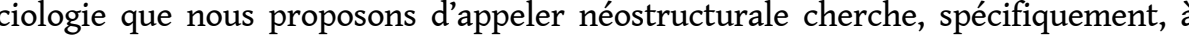
observer, modéliser, comparer ces processus sociaux et ces formes de discipline sociale. Elle part du principe que les comportements sont largement déterminés par les appartenances catégorielles plus ou moins conflictuelles, d'une part ; et par les systèmes d'interdépendances économiques et symboliques entre acteurs en opposition, d'autre part. Les acteurs individuels ou organisationnels ont à la fois des intérêts divergents et des relations d'interdépendance multiples et multilatérales. Sur le court terme, ils choisissent dans une certaine mesure leurs relations; sur le long terme, ils sont construits - entre autres - par les relations qu'ils ont plus ou moins choisies (White, 1992). Le terme «structural » se réfère ici à des régularités observées dans les relations d'échanges socio-économiques multiples, formelles et informelles, entre membres ${ }^{4}$. Les interactions et relations d'échange stables et durables représentent des interdépendances multilatérales en matière d'engagement (moral, symbolique) et d'accès aux ressources. Elles s'agrègent et se combinent en une trame de liens réguliers, en une structure relationnelle synonyme d'opportunités et de contraintes spécifiques à chaque contexte organisé de niveau méso. Les processus sociaux fondamentaux de la vie sociale à cette échelle, évoqués plus haut (solidarités, socialisations, contrôles, institutionnalisations) sont, en partie, le produit de ces régularités construites dans la gestion des interdépendances entre acteurs en conflit ou en concurrence, utilisant les organisations formelles comme outils. Sans une telle approche dynamique des interdépendances de ressources et des engagements (qui définissent les relations sociales), il est difficile de comprendre les mécanismes sociaux génériques à l'œuvre dans toute forme d'action collective. Ces processus n'existent que parce qu'ils peuvent s'appuyer sur des formes structurales et parce qu'ils interagissent entre eux.

14 Ainsi, un fait social doit être observé à plusieurs niveaux d'action analytiquement différents, ce qui rend inséparable l'analyse des relations individuelles de celle de relations organisationnelles. Pour rendre compte de cette complexité verticale du monde social contenu dans la cohabitation de plusieurs niveaux, il faut articuler ces niveaux et leurs dynamiques, propres et en coévolution. La sociologie de la société organisationnelle peine encore à analyser de manière généralisable les modalités contemporaines de construction du niveau macro par ces acteurs et processus de niveau méso. La perspective ouverte par la sociologie néostructurale est celle d'une connaissance de la société organisationnelle et marchande au travers d'une étude de la dynamique des systèmes d'interdépendances superposés, multiniveaux et partiellement emboîtés, interorganisationnels et interindividuels. Cette approche des interactions entre le global et le local n'en est qu'à ses débuts ${ }^{5}$.

La connaissance des formes de discipline sociale reconnues comme légitimes par les membres des collectifs est le résultat d'un travail permanent d'évaluation de la pertinence et de l'acceptabilité des comportements. Mais c'est notamment dans et à travers la vie relationnelle que ces formes sont identifiées, mesurées, éprouvées, acceptées ou rejetées. En conséquence, les jugements de légitimité dépendent fortement, 
eux aussi, de la connaissance des appareils organisationnels mis en place pour rendre possible l'action collective organisée. Les jugements de pertinence sont au cœur de la rationalité sociale et de la discipline sociale : ils créent un lien complexe entre l'individu et la société en ceci qu'ils permettent aux individus de contextualiser leurs comportements et ceux d'autrui, puis de chercher à peser sur la restructuration de ces contextes et le déploiement de ces processus si ces contextes apparaissent comme des structures d'opportunités et de contrainte soutenant l'action commune. Créer une discipline sociale repose sur la stimulation d'une rationalité sociale ${ }^{6}$ sans laquelle les processus sociaux ${ }^{7}$ fondamentaux rendant possible l'action collective et la résolution de ses dilemmes n'ont pas de sens pour les acteurs et ne peuvent pas se déployer. La sociologie néostructurale contemporaine conçoit ainsi la coconstitution et la coévolution du sujet et de la structure grâce à l'endogénéisation de la structure dans la rationalité sociale des acteurs et grâce aux conséquences de cette endogénéisation dans l'attribution de sens aux comportements, dans la conception de stratégies adaptées à un état donné de la structure. La structure sociale n'est émergence des interactions entre les individus que si l'on reconnaît que ces interactions sont elles-mêmes déjà conditionnées de manière récursive par les structures établies de la société organisationnelle.

Face à des défis environnementaux, technologiques et éthiques sans précédent, les formes émergentes de coordination de l'action collective organisée sont de plus en plus complexes et soumises à des exigences d'adaptation rapide. Chacun peut voir que les formes de discipline sociale que les citoyens acceptent ou non comme légitimes évoluent avec les changements technologiques (eux-mêmes induits par les ambitions de pouvoir et de contrôle sur l'environnement naturel et sur les autres). Les moyens de transport physiques ont modifié les formes de solidarité locale - puisque partir et revenir, s'éloigner et se rapprocher, deviennent plus facile. Les communications à distance sont plus rapides et plus faciles avec l'internet, mais aussi les formes de contrôle social avec l'étau de la surveillance, en partie volontaire, qui se resserre sur les pratiques et relations des individus - sur celles des plus faibles davantage que sur celles des plus forts. Les techniques de contrôle des comportements individuels par la manipulation des réactions sensorielles et des émotions atteignent une sophistication qui permet aux organisations d'intervenir directement sur ces comportements plutôt que sur leurs déterminants. La généralisation de la compétition, la flexibilisation des marchés du travail, les mobilisations de la société civile poussent les acteurs à chercher ou créer des instruments nouveaux de gestion de leurs interdépendances économiques et sociales. L'émergence des « réseaux» digitaux, personnels et organisationnels, ainsi que des plates-formes informatiques permettant leur organisation créent un contexte où les interdépendances peuvent constituer un capital relationnel pour l'individu ou un capital social pour l'organisation aux effets parfois vertueux, parfois destructeurs.

\section{Oppositions, interdépendances et dynamiques des structures multiniveaux}

La société organisationnelle est une société de classes dans laquelle les acteurs individuels et collectifs, gagnants, perdants et accapareurs d'opportunités, maîtrisent de manières très variables la propriété et le contrôle des appareils organisationnels et les formes de discipline sociale sous-jacents au processus sociaux. Les individus et organisations y opèrent nécessairement dans des contextes multiniveaux qui se restructurent en 
permanence à l'échelle mésosociale. C'est à travers cette dynamique des structures de contrainte et d'opportunités multiniveaux, superposées, que l'approche néostructurale permet de modéliser conjointement oppositions et interdépendances ${ }^{8}$.

En effet, à l'échelle méso, les structures d'opportunité et de contrainte ne sont pas des contextes multiniveaux statiques, définitivement stabilisés. Par exemple, dans l'examen de cette concurrence régulatoire qui caractérise les processus d'institutionnalisation, la connaissance de la dynamique des systèmes d'interdépendances multiniveaux, des coûts et des bénéfices de la synchronisation entre ces dynamiques, est une connaissance des plus complexes et difficiles à construire. Elle est cependant indispensable pour comprendre comment les acteurs sociaux construisent aujourd'hui le niveau macrosocial. Les modèles néostructuraux, inspirés de ceux proposés par White et al. (1976; voir aussi Breiger, 1990), sont proches des acteurs, de leurs relations d'interdépendances, de leurs positions et des relations d'interdépendances entre ces positions. Ils sont donc particulièrement adaptés pour l'exploration du niveau mésosocial et de sa dimension intrinsèquement multiniveaux. La construction des relations entre organisations contribue à reconfigurer l'architecture institutionnelle des sociétés. Cela signifie, par exemple, que cette reconfiguration ne peut se centrer exclusivement sur l'étude des élites individuelles et de leurs réseaux personnels. Il s'agit plutôt de situer ces individus et ces élites dans des structures mésosociales. Les méthodes d'analyse organisationnelle et structurale sont très utiles pour examiner les relations entre organisations et leur environnement comme clé de l'institutionnalisation, pour apporter un éclairage pertinent sur les systèmes d'action qu'elles constituent « ensemble " ${ }^{9}$.

19 Empiriquement, développer la connaissance contemporaine de ce niveau mésosocial s'appuie donc sur un programme de recherche centré sur la coévolution des systèmes d'interdépendances des individus et des organisations, à des «étages » différents de la réalité sociale. Cette coévolution est en effet peu connue: quels sont les effets de l'évolution d'un niveau sur l'évolution d'un autre? Quelles contraintes de synchronisation de ces évolutions existent dans la réalité économique et sociale? Si différentes formes de synchronisation existent, qui en paie le coût: les exclus désorganisés, les classes moyennes accapareuses d'opportunités, les élites au pouvoir ? La synchronisation des évolutions propres à chaque niveau de réalité sociale se fait par exemple dans les ajustements relationnels requis par les mobilités et les parcours professionnels. On peut alors faire l'hypothèse que cette synchronisation se fait en partie par le fonctionnement contemporain des marchés du travail flexibilisés où les coûts et bénéfices d'adaptation des entreprises aux individus, le plus souvent des individus aux entreprises, sont plus facilement transférables aux plus faibles. Ces adaptations et leurs coûts invisibles, presque toujours à la charge des individus, rarement à celle de l'organisation, sont encore mal mesurés.

20 Pour mettre au jour ces dynamiques des structures multiniveaux, l'utilisation de méthodes d'analyse organisationnelle qualitative et quantitative est primordiale pour la sociologie. Ces méthodes ne privilégient pas les approches micro et macro et ne se centrent pas sur le passage direct de l'un à l'autre par agrégation mécanique. Elles permettent la conceptualisation, les mesures et les modélisations du niveau mésosocial pour mieux comprendre la forme que prennent les processus.

21 C'est à la lumière de cette problématique qu'il faut envisager l'épistémologie des analyses de réseaux sociaux et organisationnels qui émergent en sociologie néostructurale à partir du travail de Harrison White et de ses étudiants dans les années 1970. Ce que ces analyses 
rendent visibles et permettent de mesurer ce sont précisément ces formes de discipline sociale évoquées plus haut. Les réseaux ne sont pas des acteurs collectifs; ce sont des artefacts de méthode qui mesurent les traces des processus sociaux fondamentaux examinés sous l'angle de leurs rouages relationnels. La connaissance (reconstitution, analyse et modélisation, visualisation, etc.) de ces réseaux de toutes natures est en plein développement scientifique et exploitation managériale. Nous avons montré que le suivi de ce développement est un enjeu crucial pour la sociologie, en particulier lorsque ces développements sont contemporains d'autres phénomènes comme la manipulation des émotions collectives et l'industrialisation des corps.

Quel est le rôle de la sociologie dans ce cadre? Au niveau le plus élevé de généralité, la sociologie est un ensemble de pratiques d'observation, d'analyse et de synthèse qui se veulent méthodiques, voire scientifiques, de mise au jour, de diffusion, d'alerte ou de dénonciation autour de ces structures, de ces disciplines sociales, de ces pouvoirs, de leur exercice et des conséquences à l'échelle agrégée ou désagrégée des décisions des acteurs qui représentent ces pouvoirs. Depuis ses débuts au XIX siècle, la sociologie a ajouté à ses approches organisationnelles des analyses relationnelles de la vie en société. Weber et Simmel sont parmi les auteurs les plus connus de cet enrichissement de la réflexion politique qui articulent l'analyse de la discipline sociale à la réflexion sur le droit positif et sa mise en œuvre.

Avec des bases de données comme celles de "big surveillance ", le travail que les sociologues font à petite échelle devient possible à grande échelle (pour ceux qui contrôlent l'accès aux données). Ce n'est pas seulement l'identification de « communautés » mais l'analyse de leurs cultures, mobilisations et fonctionnements qui deviennent possible. Le fait que les grands réseaux pauvres deviennent de grands réseaux riches modifie aussi la valeur ajoutée des sociologues à la compréhension de la société organisationnelle. Dans le contexte où les élites politiques et économiques, celles qui contrôlent les plus grands appareils organisationnels, disposent d'un nouvel avantage dans la connaissance des processus sociaux génériques de la société moderne, le rôle de la sociologie est de chercher à rendre ces processus visibles pour tous. Pour les sociologues aussi, se donner les moyens d'une moins grande opacité de la société organisationnelle n'est pas la moindre des ambitions. La transdisciplinarité devient une question de survie disciplinaire.

\section{La lutte pour la connaissance du niveau mésosocial}

La relation entre sociologie et sciences de la complexité ne peut pas être indépendante de ce contexte nouveau. Les méthodes sociologiques - et la sociologie elle-même - évoluent avec la place nouvelle que prend cet ensemble de problématiques. Articulées aux analyses des formes d'action collective organisées, ces analyses de réseaux renouvellent la connaissance en particulier du niveau mésosocial, c'est-à-dire des formes de structuration et d'exercice du pouvoir sur lesquels les jugements de légitimité - souvent exclusivement formatés par des analyses macrosociologiques - peinaient à trouver prise. Plus les citoyens utilisent les analyses organisationnelles et de réseaux pour évaluer la légitimité des formes de discipline sociale qui leur sont imposées ou qu'ils s'imposent aussi eux-mêmes, plus la sociologie fait son travail et justifie son existence. Ce qu'il faut éviter c'est que la connaissance des structures d'opportunité et de contrainte apportée par ces méthodes, surtout dans le contexte de «big surveillance», soit trop 
asymétriquement distribuée, qu'elle n'appartienne qu'aux grandes institutions policières ou militaires ou aux grandes entreprises privées.

La statistique de réseaux est une composante importante - quoique encore trop peu développée - de la méthode structurale. Par exemple, expliquer les différences ou inégalités dans l'accès à des ressources requiert un examen de ce qui détermine les choix sociométriques opérés par les acteurs, notamment les attributs de ces acteurs - que ces attributs soient micro, méso ou macrosociaux. Le réel savoir-faire sociologique en analyse de réseaux sociaux consiste à travailler conjointement en bases acteurs (individus, organisations, etc.), en bases relations et dans l'articulation des deux grâce à la rationalité sociale des acteurs. Or, dans la mesure où la connaissance des processus sociaux suppose une observation de la coévolution des comportements et des relations, l'utilisation de données relationnelles comme variables dépendantes dans des modèles statistiques pose des problèmes spécifiques.

C'est le cas lorsque l'on cherche à expliquer pourquoi certains acteurs ont plus facilement accès à davantage de ressources, ou à relever les critères qu'ils utilisent pour choisir ou trier leurs relations, ou à expliquer l'absence de réciprocité dans les échanges observés, etc. Cela revient à se demander quelle est l'influence de plusieurs dimensions formelles de la structure d'un système social (origine sociale, niveau d'éducation, statut, appartenances associatives, spécialité, lieu de travail, genre, autres attributs) sur les choix relationnels observés. Dans une organisation, la structure formelle, destinée à coordonner l'action collective, reflète et reproduit aussi des différences et des inégalités propres au terrain étudié. En particulier, elle offre ou non aux membres l'occasion de profiter individuellement de leur position ou de se créer de nouvelles relations ${ }^{10}$.

Ces modèles prennent en compte, quoique partiellement, l'interdépendance entre les relations partant de, et parvenant à, un acteur, ainsi que la dépendance entre les relations constituant une dyade, une triade ou une autre sous-structure. Quant aux variables indépendantes ou explicatives, elles sont les caractéristiques des acteurs et des relations (au temps précédent, par exemple, dans les modèles longitudinaux), ou des caractéristiques dérivées de leur position dans la structure formelle de leur système social (en particulier, diverses formes de similitudes entre eux). La collecte de données de ce type, coûteuse, difficile, reste néanmoins incontournable si les sociologues veulent pouvoir modéliser, comparer et mieux connaitre les processus sociaux génériques de la vie sociale. . Les outils d'analyse doivent alors être reconsidérés en prenant en compte les avancées de l'algorithmique afin qu'ils puissent «passer à l'échelle » (Cardon et Prieur, 2007).

28 C'est à ce stade que se pose la question de la nature du dialogue entre la sociologie néostructurale plus familière de l'étude des petits réseaux riches avec les disciplines engagées actuellement dans l'analyse des « systèmes complexes ». S'il ne fait aucun doute qu'à ce niveau de généralité, des réseaux sociaux et des réseaux métaboliques peuvent partager des propriétés formelles communes, par exemple l'hétérogénéité et la structure de "petits mondes", entre les interactions microscopiques et le comportement global, la structure de niveau méso est très peu prise en compte dans la modélisation, alors que c'est elle qui intéresse le plus les sociologues centrés sur une statistique des petits réseaux multiniveaux caractéristiques de la société organisationnelle et marchande. La conception des méthodes qui permettent aux sciences de la nature et aux sciences sociales de se développer conjointement en est encore à un stade très exploratoire. Mais 
surtout les épistémologies des uns et des autres sont très différentes, à commencer par celles des spécialistes des grands réseaux et de la complexité ainsi définie entre eux.

\section{Vers une épistémologie commune avec les sciences de la complexité ?}

Depuis l'émergence des démocraties occidentales, les citoyens de ces sociétés avaient appris, au moins minimalement, à surveiller les pouvoirs qui décident de leurs destinées et de leurs conditions de vie, à tous les niveaux - des plus locaux jusqu'aux plus globaux où ils pouvaient définir et défendre leurs intérêts. Cette contre-surveillance s'appuie en partie sur l'évaluation de la légitimité des structures institutionnelles et formelles dans lesquelles ces pouvoirs sont distribués et exercés. Depuis longtemps cependant, la seule connaissance de ces structures formelles, de leur fonctionnement et des conséquences des décisions qui y sont prises n'est jamais apparue comme suffisante pour cette évaluation. Les acteurs qui exercent des pouvoirs de toutes sortes peuvent mettre en place des appareils formels en apparence parfaitement transparents et démocratiques, mais en réalité, souvent, opaques, monocratiques, autoritaires, parfois des "usines à gaz " échappant à toute surveillance bottom up. Ce caractère souvent trompeur des appareils formels tient au fait que les structures d'opportunité et de contraintes sociales sont des systèmes encore bien plus complexes que les organigrammes. Même si les appareils formels sont l'ossature des structures, les secondes sont moins lisibles que les premiers. Pour les évaluer, les citoyens cherchent les réalités sociales sous-jacentes et des critères permettant de défendre leurs intérêts dans les batailles de légitimité. Dans le contexte de cette société organisationnelle, «big surveillance " renforce l'asymétrie habituelle : les élites peuvent voir au travers de la société civile, identifier et localiser les formes d'action collective organisée et leurs processus sociaux, alors que l'inverse est encore moins évident qu'il ne l'a jamais été.

La connaissance de la dimension mésosociale est très développée en sociologie. Les analyses sociologiques décrivent et comparent les processus fondamentaux de l'action collective sous leur angle relationnel et organisationnel. Elles proposent une sociologie "néostructurale » distincte des formes de structuralisme où l'action individuelle ne comptait pas plus que les formes très diversifiées et complexes d'action collective organisée. L'objectif de cette sociologie est de rendre plus lisibles - en les modélisant et en les comparant - les formes de discipline sociale pour enrichir le débat sur leur légitimité, celles des structures sous-jacentes, des formes contemporaines de pouvoirs et de leur exercice. L'accent est mis ici sur le niveau méso, sur la modélisation des mécanismes sociaux qui engendrent les effets mesurés à différentes échelles. Cela ne disqualifie en rien les analyses agrégées au niveau macro. Mais ce qui manque encore à la sociologie, c'est la connaissance systématique de la relation de cette dimension mésosociale avec la macrosociologie, sa capacité à suivre - avec des méthodes adaptées les efforts - réussis ou non - de construction et de stabilisation du niveau macro sans présupposer un système politique rigide qui réussit toujours cette construction en intégrant parfaitement tous ses sous-systèmes (comme le faisait le structurofonctionnalisme). L'ambition est ainsi d'enrichir les moyens de la sociologie et ce faisant de donner de nouvelles prises aux citoyens dans leurs jugements de pertinence et de légitimité. Ce jugement n'est pas simplement rhétorique. Il a des effets et des conséquences. 
31 Pour enrichir le regard macrosocial en articulant les niveaux méso et macro grâce à la réflexion contemporaine sur les systèmes complexes, la possibilité du dialogue heuristique tient précisément au fait que de nombreux sociologues, physiciens, mathématiciens, informaticiens, biologistes, etc. acceptent l'idée qu'une épistémologie commune reste à construire. Techniques méthodologiques et épistémologies ayant toujours coévolué, la question revient dès lors de savoir quelle serait cette épistémologie commune pour que ce dialogue puisse être heuristique ? Comment réfléchir ensemble à l'articulation du niveau mésosocial et du niveau macrosocial, à l'articulation des petits réseaux riches et des grands réseaux pauvres (dont on peut essayer de connaître, pour commencer, les modalités de construction) ? Les sociologues ne peuvent collecter des données et reconstituer des structures et des processus sociaux en ignorant que les acteurs sociaux sont capables de donner un sens à leur action, de gérer leurs interdépendances, d'endogénéiser la structure d'opportunités et de contraintes, de politiser leurs échanges. Les acteurs sociaux agissent de manière réflexive. Les règles qu'ils se donnent et qui contribuent à produire des régularités dans leurs comportements, sont produites dans des contextes qu'ils construisent en hiérarchisant leurs identités et groupes de référence, dans les cultures et dans les droits dont ils héritent et qu'ils recréent, dans des négociations menées par des représentants eux-mêmes engagés dans des rapports de forces propres à leurs systèmes politiques et administratifs. Ainsi la question de la légitimité des règles et des structures, du sens et de la pertinence de l'action pour les acteurs sociaux, posée plus haut au départ de cette réflexion, est fondamentale pour les explorations empiriques heuristiques et pour la recherche de cette épistémologie. Car l'objectif de la sociologie est de proposer un regard et des analyses pertinentes pour la compréhension des capacités d'action collective - mais aussi des impuissances d'action collective - promouvant des changements institutionnels, et plus généralement des changements de civilisation, qui semblent requis par beaucoup de défis (notamment écologiques) contemporains.

La sociologie a intérêt à ce que cette épistémologie commune émerge. En effet les enjeux sociaux et politiques des phénomènes que nous observons sont tels que les sociologues perdent le semblant de priorité dont ils croyaient bénéficier au cours du $\mathrm{xx}^{\mathrm{e}}$ siècle sur l'analyse et l'observation, dites scientifiques, des formes de discipline sociale. Dans la pratique, les sociologues n'ont jamais eu de monopole académique. Par exemple, pratiquement, dès le début des années 1960, les économistes commençaient à défier les approches sociodémographiques dans le domaine du choix conjoint en modélisant à leur manière le "marché du mariage ». Aujourd'hui le semblant de monopole est remis en question aussi bien par les économistes, épidémiologues, physiciens-statisticiens et informaticiens, neurobiologistes et spécialistes du marketing que par la police, l'armée, les cabinets de consultants (en «intelligence économique», en «community management» dans les entreprises et les administrations). Face à ces enjeux de développement technologique et de contrôle social, s'ils veulent continuer à porter un regard critique et pertinent sur les formes de l'action collective et des disciplines sociales considérées comme légitimes, voire d'être utiles à la société civile et au débat public, les sociologues doivent diversifier les types de données relationnelles et de formalismes - en provenance notamment de la communauté dite des «systèmes complexes »-, valoriser leur diversité plutôt que de l'ignorer (Prieur et al., 2008).

L'objet «réseau» est particulièrement transdisciplinaire. L'analyse des systèmes d'interdépendances entre acteurs est un bon point de départ pour cette recherche 
d'épistémologie commune. L'art de la description et de l'analyse (recherche de structure) dans les réseaux de toutes sortes a besoin de cette diversité. Ne serait-ce que pour mieux exploiter la quantité phénoménale de données de réseaux de plus en plus disponibles - via l'internet ou d'autres sources - pour l'analyse secondaire. Les formalismes permettant la modélisation des structures relationnelles à toutes les échelles se diversifient et s'enrichissent à très grande vitesse. Cette diversité est une richesse si les langages de formalisation se traduisent, se complètent, se font mutuellement évoluer ou permettent de spécifier leurs limites respectives. La concurrence entre eux existe au sens où ce qu'exposent certains modèles est laissé dans l'ombre par d'autres, mais cette concurrence est limitée par le fait que la seule manière de mettre au service des citoyens et citoyennes les instruments d'analyse et d'aide à l'évaluation de la légitimité des structures et des formes de disciplines sociales, c'est d'enrichir et diversifier les boîtes à outils méthodologiques. La technologie, sa connaissance et ses usages sont ici un enjeu majeur.

La survie de la sociologie, notamment, dépend en partie de sa capacité à poursuivre l'enrichissement de ses approches pour adapter ses capacités à l'analyse systématique des données dont la composition et la structure deviennent de plus en plus diversifiées et complexes. On y combine des données d'observation ethnographique, des données d'analyse secondaire de fichiers statistiques, des analyses de discours et d'entretiens, des données de questionnaires et d'informations construites «en ligne"-accessibles notamment du fait de l'essor d'internet et de la constitution de bases par datamining. Cet enrichissement des approches peut correspondre parfois au développement de méthodes qui relèvent de formes plus ou moins créatives d'arte povera. L'enjeu pour la sociologie est alors de trouver les moyens de continuer à renouveler ses méthodes au contact des approches multidisciplinaires de la complexité pour éviter de devenir une technique presse-bouton fabriquant des cartes en couleur à partir de données collectées «à l'aveugle" sans que ces dernières n'apportent aucun éclairage sur les acteurs et les processus, aucune lisibilité accrue des structures et des pouvoirs - et donc aucune aide à l'évaluation de la légitimité des formes de discipline sociale émergentes.

\section{BIBLIOGRAPHIE}

Barabási, A.-L., Linked : How Everything Is Connected to Everything Else and What It Means for Business, Science, and Everyday Life, New York, Plume Books, 2003.

Brailly, J. et Lazega, E., « Diversité des approches de la modélisation multiniveaux en analyses de réseaux sociaux et organisationnels ", à paraître, Mathématiques et Sciences humaines, 2012.

Breiger, R. L. (éd.), Social Mobility and Social Structure, Cambridge, Cambridge University Press, 1990.

Bryk, A. S., Raudenbush, S. W., Hierarchical linear models, Londres, Sage, 1992. 
Cardon, D. et Prieur, C., « Les réseaux de relations sur internet : un objet de recherche pour l'informatique et les sciences sociales » in C. Brossaud et B. Reber (dir.), Humanités numériques 1. Nouvelles technologies cognitives et épistémologie, Paris, Lavoisier, 2007.

Chavalarias, D., « Metamimetic Games : Modeling Metadynamics in Social Cognition », Journal of Artificial Societies and Social Simulation, vol. 9, $\mathrm{n}^{\circ}$ 2, http://jasss.soc.surrey.ac.uk/9/2/5.html

Cointet, J.-Ph. et Roth, C., « How Realistic Should Knowledge Diffusion Models Be ? » Journal of Artificial Societies and Social Simulation, vol. 10, n³, 2007, p. 5, http:// jasss.soc.surrey.ac.uk/10/3/5.html

DiMaggio, P., « Structural analysis of organizational fields », in B. Staw et L.L. Cummings, Annual Review of Sociology, vol. 13, 1986, p. 243-282.

Duijn, M.A.J. van, Snijders, T.A.B. et Zijlstra, B.J.H., « P2 : a random effects model with covariates for directed graphs », Statistica Neerlandica, vol. 58, 2004, p. 234-254.

Frank, O., « Random Sampling and Social Networks : A Survey of Various Approaches ", Mathématiques, informatique et sciences humaines, vol. 26, 1988, p. 19-33.

Frank, O. et Strauss, D., « Markov Graphs », Journal of the American Statistical Association, 81, 1986, p. 832-842.

Guillaume, J.-L. et Latapy, M., « Relevance of massively distributed explorations of the internet topology: simulation results », in IEEE Infocom, 2005.

Holland, P. W. et Leinhardt, S., « An exponential Family of Probability Distributions for Directed Graphs (with discussion) », Journal of the American Statistical Association, vol. 76, 1981, p. 33-65.

Krackhardt, D., « Predicting with Networks : Nonparametric Multiple Regression Analysis of Dyadic Data », Social Networks, vol. 10, 1988, p. 359-381.

Latapy, M., Magnien, C. et Del Vecchio, N., « Basic Notions for the Analysis of Large Two-mode Networks », Social Networks, vol. 30, 2008, p. 31-48.

Lazega, E., « Rationalité, discipline sociale et structure », Revue française de sociologie, vol. 44, 2003, p. 305-330.

-, «Capital social, processus sociaux et capacité d'action collective », in A. Bevort et M.

Lallement (éd.), Capital social : Échanges, réciprocité, équité, Paris, La Découverte, 2006, p. 213-225.

_, « Pertinence et structure », Revue suisse de sociologie, vol. 37, 2011, p. 127-149.

-, « Sociologie néo-structurale », in R. Keucheyan et G. Bronner (éd.), Introduction à la théorie sociale contemporaine, Paris, PUF, 2012.

Lazega, E., Jourda, M.-T., Mounier, L. et Stofer, R., « Des poissons et des mares : l'analyse de réseaux multi-niveaux ", Revue française de sociologie, vol. 48, 2007, p. 93-131.

Lazega, E., Jourda, M., Mounier, L., Brailly, J., Bruna, M.-G., Chatellet, J., Favre, G., Penalva, E. et Pina-Stranger, Á., « Dynamics of multilevel networks with two levels of agency », Communication présentée à la conférence de l'observatoire des réseaux intra- et interorganisationnels « Research on the Organizational Society : Advances in Multilevel and Dynamic Network Analysis », Dauphine, 16 juin 2011.

Pattison, Ph. et Robins, G. L., « Building models for social space : Neighbourhood based models for social networks and affiliation structures ", Mathematiques et science humaines, vol. 4, 2004, p. 11-29.

Perrow, Ch., «A Society of Organizations », Theory and Society, vol. 20, 1991, p.725-762. 
Prieur, C., Cardon, D., Beuscart, J.-S., Pissard, N. et Pons, P., « The stength of weak cooperation : A case study on flickr », 2008, http://arxiv.org/abs/0802.2317

Robins, G. L., Woolcock, J. et Pattison, P., « Small and other worlds: Global network structures from local processes ", American Journal of Sociology, vol. 110, 2005, p. 894-936.

Rouchier, J., Lazega, E. et Mounier, L., « Articulation of hierarchy and networks as an evolving social structure », in T. Terano, H. Kita, H. Deguchi et K. Kijima (éd.), Agent-Based Approaches in Economic and Social Complex Systems IV, Springer, 2007, p. 97-104.

Snijders T. A. B., « Methods for longitudinal social network data », in E. .M. Tiit, T. Kollo et H. Niemi (éd.), New Trends in Probability and Statistics, vol. 3, Utrecht, Hollande, vsP, 1995, p. 21-227.

-, « Models for longitudinal network data », in P. Carrington, J. Scott et S. Wasserman (éd.), "Models and Methods in Social Network Analysis », chap. 11, New York, Cambridge University Press, 2005.

Snijders, T. A. B. et Van Duijn, M. A. J., « Simulation for Statistical Inference in Dynamic Network Models ", Simulating social phenomena, R. Conte, R. Hegselmann et P. Terna (éd.), Berlin, Springer, 1997, p. 493-512.

Snijders, T. A.B., Steglich, C. E.G. et Schweinberger, M., « Modeling the co-evolution of networks and behaviour ", in K. van Montfort, H. Oud et A. Satorra (éd.), Longitudinal models in the behavioral and related sciences, Routledge, 2006.

Snijders, T. A.B. et Bosker, R., Multilevel Analysis, Londres, Sage, 1999.

Tarissan, F., Latapy, M. et Prieur, C., Efficient Measurement of Complex Networks Using Link Queries. Proceedings of the IEEE International Workshop on Network Science For Communication Networks (NetSciCom'09), 2009.

Wang, P., Robins, G., Pattison, P. et Lazega, E., « Exponential random graph models for multilevel networks », Social Networks, vol. 35, 2013, p. 96-115.

White, H. C., Boorman, S. C. et Breiger, R. L. (1976), « Social Structure From Multiple Networks I. Blockmodels of Roles and Positions ", American Journal of Sociology, vol. 81, p. 730-80.

White, H. C., Identity and Control, Princeton, Princeton University Press, 1992.

Zijlstra, B., J.H., Duijn, M. A.J. van, et Snijders, T. A.B., « The multilevel p2 model - A random effects model for the analysis of multiple social networks ", Methodology, vol. 2, 2006, p. 42-47.

\section{NOTES}

1. Voir à ce sujet : A Comparison of the 2008 and 2012 NCTC Guidelines : http://www.fas.org/sgp/ othergov/intel/nctc_guidelines.pdf

2. Voir à ce sujet la reconstitution des discussions par l'enquête du Wall Street Journal du 13 décembre 2012 et les informations disponibles sur son site : http://online.wsj.com/article_email/ SB10001424127887324478304578171623040640006-lMyQjAxMTAyMDEwMzExNDMyWj.html Nous remercions Cyrille Amistani d'avoir attiré notre attention sur cette décision de l'Administration américaine et sur cet article.

3. Fleury et Friggeri pratiquent un calcul de communautés et de leur dynamique des mesures de la cohésion sociale dans les profils Facebook, qui commence à s'insérer dans les problématiques plus traditionnelles de l'analyse des réseaux sociaux en sciences sociales. 
4. Le néostructuralisme contemporain est différent des structuralismes français des années 1960 parce qu'il s'appuie sur des théories de l'action individuelle et collective qui n'apparaissaient pas comme pertinentes à l'époque.

5. Nous avons proposé (Lazega et al., 2007) un mode structural d'articulation de ces niveaux qui consiste à examiner séparément des systèmes d'oppositions et d'interdépendances de niveaux différents, puis à les articuler grâce à des informations systématiques sur l'appartenance de chaque individu du premier niveau (interindividuel) à l'une des organisations du second niveau (interorganisationnel). Cette approche est généralisable à $n$ niveaux.

6. Voir Lazega $(2003,2011)$ au sujet de la spécificité de cette rationalité sociale.

7. Pour une approche du capital social comme ensemble des processus relationnels constituant une capacité d'action collective, voir Lazega (2006).

8. Particulièrement bien adaptés à l'étude du niveau mésosocial et des effets de contextes dans les milieux formellement organisés, les modèles statistiques multiniveaux classiques (Bryk et Raudenbush, 1992 ; Snijders et Bosker, 1999) ont connu un fort développement au cours des trente dernières années. Ces modèles ont beaucoup aidé à intégrer les individus, les groupes et organisations auxquels ils appartiennent comme unités d'analyse. Pour leur part, les modèles d'analyse de réseaux sociaux et organisationnels ont également connu un développement rapide, permettant de modéliser des systèmes relationnels et leur évolution. Mais la réalité sociale que nous observons est le plus souvent à la fois multiniveaux, dynamique et relationnelle. Dans la mesure où chaque niveau constitue un système d'action, d'interactions et d'échange de ressources différentes, il est aussi nécessaire aujourd'hui de développer une approche statistique pouvant les examiner conjointement. Sur le plan méthodologique, la constitution de corpus multiniveaux et longitudinaux et leur analyse sont au cœur des recherches empiriques néostructurales. Voir à ce sujet Snijders (1995 et travaux ultérieurs) ou les travaux de Pattison et al., (2004; Robbins et al., 2005). La question de savoir si la structure d'un collectif reste stable malgré un fort turnover de ses membres et un fort turnover des relations entre ces membres est ainsi une question classique de la sociologie (Lazega et al., 2011; Wang et al., 2013 ; Brailly et Lazega, 2012).

9. Voir à ce sujet la notion d'« organizational field» de DiMaggio (1986).

10. Vérifier des hypothèses d'influences sur les choix relationnels pose le problème de l'indépendance des observations. Le fait de choisir Pierre comme ami n'est pas nécessairement indépendant du fait de choisir également Paul, les deux pouvant eux-mêmes être des amis. Une relation engage donc au moins trois individus. Cela conduit à toutes sortes de dépendances entre les observations (voir par exemple Frank et Strauss, 1986; Krackhardt, 1988; Snijders, 1995 ; Snijders et Van Duijn, 1997 ; Snijders et al., 2006 ; Pattison et Wasserman, 1999, Zijlstra et al., 2006). Ces dépendances compliquent l'analyse statistique des relations sans qu'un modèle probabiliste général (i.e. une définition fondamentale de ce qu'est un réseau " au hasard ») n'ait encore été trouvé - s'il existe - pour maîtriser de manière tout à fait fiable les inférences dans de telles conditions (en particulier des petites populations). Cependant, des modèles spécifiques et plus limités ont été développés avec une conception de l'inférence différente de celle de la statistique macrosociologique à laquelle les sociologues sont le plus souvent habitués (Snijders et al., 2006). Voir à ce sujet la littérature qui part du modèle $p 1$ de Holland et Leinhardt (1981) pour passer par le modèle $p 2$ (Duijn et al., 2004 ; Snijders et Van Duijn, 1997), puis $p^{*}$ actuellement développé sous le nom d'ERGM (exponential random graph models) (Robins et al., 2005). 


\section{RÉSUMÉS}

L'émergence de bases de données à la fois très grandes et très riches (dont l'existence-même est souvent révélée par les scandales de big surveillance liés aux nouvelles technologies de contrôle social capables de zoomer de très loin sur les acteurs individuels et collectifs) oblige les sciences sociales et celles des systèmes complexes à combiner leurs efforts et à dépasser l'opposition confortable entre petits réseaux riches et grands réseaux pauvres - une opposition qui leur permettaient de se maintenir mutuellement à distance. Pour cela, l'un des défis que les deux disciplines doivent relever est une réflexion commune sur les théories de l'action et présupposés comportementaux qui guident leurs analyses séparées et qui doivent orienter leurs analyses conjointes. La sociologie néostructurale propose une approche qui peut aider à cette réflexion en se centrant sur le niveau mésosocial, les processus et structures dynamiques et multiniveaux, c'est-à-dire sur les formes spécifiques de discipline sociale qui caractérisent ce niveau méso, et la manière dont les acteurs évaluent la légitimité de ces formes pour leur donner un sens.

The emergence of very large and very rich network datasets (often revealed by big surveillance scandals related to new technologies of social control that are able to zoom in and out on individuals and collective actors) forces the social sciences and complexity sciences to join strengths, beyond the old separation between small and rich network datasets and large and poor network datasets. One of the challenges that both disciplines face is to reassess the behavioural assumptions on which their joint analyses should be based. Neo-structural sociology offers an approach that can help in this reassessment by focusing on meso-level, dynamic and multilevel structures and processes, i.e. on the meso-level of society, its specific forms of social disciplines, and the ways in which actors evaluate the legitimacy of these forms to make sense of them.

\section{INDEX}

Mots-clés : sociologie néostructurale, disciplines sociales, processus sociaux, grands réseaux riches, big data, structures mésosociales, systèmes complexes

Keywords : neo-structural sociology, social discipline, social processes, large , meso-social structures, complex systems

\section{AUTEURS}

\section{EMMANUEL LAZEGA}

Professeur des universités à l'Institut d'études politiques de Paris (Centre de sociologie des organisations).

Parmi les publications :

« Pertinence et structure », Revue suisse de sociologie, vol. 37, 2011, p. 127-149.

Avec L. Mounier, T. Snijders et P. Tubaro, « Norms, status and the dynamics of advice networks », 
Social Networks, vol. 34, 2012, p. 323-332.

« Analyses de réseaux et classes sociales », Revue française de socio-économie, $\mathrm{n}^{\circ}$ 10, 2012,

p. 273-279.

«Sociologie néo-structurale », in R. Keucheyan et G. Bronner (éd.), Introduction à la théorie sociale contemporaine, Paris, PUF, 2012.

Avec M.-T. Jourda, et L. Mounier, « Network Lift from Dual Alters : Extended Opportunity Structures from a Multilevel and Structural Perspective », European Sociological Review, $1^{\text {re éd. en }}$ ligne 24 avril 2013, DOI: 10.1093/esr/jct002.

"Network analysis in the "Morphogenetic Society" project : A neo-structural exploration and illustration », in M. S. Archer (éd.), Social Morphogenesis, Springer, 2013, p. 167-186.

\section{CHRISTOPHE PRIEUR}

Maître de conférences à l'université Paris 7 (Laboratoire d'informatique algorithmique :

fondements et applications).

Parmi les publications :

Avec D. Cardon, «Les réseaux de liens sur internet, un outil de recherche pour l'informatique et les sciences sociales ", in C. Brossaud et B. Reber (éd.), Humanités Numériques, vol. 1, Hermès, 2007. Avec R. Lambiotte, V. Blondel, C. de Kerchove, E. Huens, Z. Smoreda et P. Van Dooren, «Geographical dispersal of mobile communication networks », Physica A: Statistical Mechanics and its Applications, vol. 387, $\mathrm{n}^{\circ}$ 21, 2008.

Avec A. Stoica et Z. Smoreda, « Extraction de réseaux égocentrés dans un (très grand) réseau social », Bulletin de méthodologie sociologique (BMS), n 101, 2009.

Avec J.-S. Beuscart, D. Cardon et N. Pissard, « Pourquoi partager mes photos de vacances avec des inconnus? Les usages de Flickr », Réseaux, n 154, 2009.

Avec S. Raux, « Stabilité globale et diversité locale dans la dynamique des commentaires de Flickr », Technique et Science Informatique, numéro spécial « Graphes de terrain », à paraître, 2010. Avec A. Stoica et Z. Smoreda, «A local structure-based method for nodes clustering. Application to a large mobile phone social network », in Social Network Analysis and Mining, Springer, 2013. 